PROCEEDINGS OF THE

AMERICAN MATHEMATICAL SOCIETY

Volume 140, Number 2, February 2012, Pages 479-492

S 0002-9939(2011)10933-X

Article electronically published on June 16, 2011

\title{
ON THE IRREDUCIBLE COMPONENTS OF THE SINGULAR LOCUS OF $A_{g}$. II
}

\author{
V. GONZÁLEZ-AGUILERA, J. M. MUNOZ-PORRAS, AND A. G. ZAMORA
}

(Communicated by Lev Borisov)

\begin{abstract}
In our earlier paper it was proved that the singular locus of $A_{g}$ (coarse moduli space of principally polarized abelian varieties over $\mathbb{C}$ ) is expressed as the union of irreducible varieties $A_{g}(p, \alpha)$ representing abelian varieties with an order $p$ automorphism of fixed entire representation. In this paper we prove that $A_{g}(p, \alpha)$ is an irreducible component of $\operatorname{Sing} A_{g}$ if and only if for a general element of this variety its automorphism group modulo $\{ \pm 1\}, G_{+}$, satisfies the equivalent conditions: $G_{+}=\langle\alpha\rangle$ or $N_{G_{+}}(\langle\alpha\rangle)=\langle\alpha\rangle$. We illustrate how these results can be used by studying the case $g=4$ and $p=5$.
\end{abstract}

\section{INTRODUCTION}

This paper is a direct continuation of our previous works (4 , [5], 6]). The central problem is to determine the irreducible components of the singular locus of $A_{g}$, the moduli space of principally polarized abelian varieties (p.p.a.v. from now on) of dimension $g \geq 3$ over $\mathbb{C}$. The singular locus $\operatorname{Sing} A_{g}$ is well known to be the set representing abelian varieties with a nontrivial automorphism group (i.e., an automorphism group different from $\{ \pm 1\})([\underline{8},[9])$.

We denote by $X$ a p.p.a.v. and by $G=\operatorname{Aut}(X)$ the group of automorphisms of $X$, understood as the group of automorphisms of the algebraic group $X$ preserving the polarization. We also denote $G_{+}=\operatorname{Aut}_{+}(X)=\operatorname{Aut}(X) /\{ \pm 1\}$. Note that $\operatorname{Aut}(X)=\operatorname{Aut}_{+}(X) \times\{ \pm 1\}$.

In [4] it was proved that $\operatorname{Sing} A_{g}$ decomposes into irreducible subvarieties $A_{g}(p, \alpha)$ representing abelian varieties with an order $p$ automorphism and fixed integer representation when restricted to the lattice $E$ defining the abelian variety. Thus, the determination of the irreducible components of $\operatorname{Sing} A_{g}$ reduces to determining the possible inclusions $A_{g}(p, \alpha) \subset A_{g}(q, \beta)$.

The main results of this paper can be summarized as follows:

Theorem 1.1. Let $A_{g}(p, \alpha)$ be an irreducible variety associated with the odd prime order $p$ automorphism $\alpha$. Assume d $\alpha$ acts on the tangent spaces of the elements of $A_{g}(p, \alpha)$ without eigenvalues equal to 1 . Then the following are equivalent:

Received by the editors May 4, 2010 and, in revised form, September 17, 2010 and November 30, 2010.

2010 Mathematics Subject Classification. Primary 14K10, 14K22; Secondary 14D15.

The first author was partially supported by Fondecyt Grant 1080030 and UTFSM's DGIP.

The third author was partially supported by CoNaCyT Grant 25811. 
i) $A_{g}(p, \alpha)$ is an irreducible component of Sing $A_{g}$.

ii) If $[X] \in A_{g}(p, \alpha)$ is a general element, then $\operatorname{Aut}_{+}(X)=\langle\alpha\rangle$.

iii) If $[X] \in A_{g}(p, \alpha)$ is a general element, then $N_{A u t_{+}(X)}(\langle\alpha\rangle)=\langle\alpha\rangle$.

The equivalence $i) \Longleftrightarrow i$ i) is the content of Theorem 3.1 and $i i) \Longleftrightarrow$ iii) is Theorem 4.2. Note that $i i) \Rightarrow i$ ) is a consequence of our previous work (4]) and ii) $\Rightarrow$ iii) is trivial. Thus the issue is to prove the converse of these propositions.

Section 2 contains a summary of previous results that will be used in the subsequent arguments as well as a couple of generalizations. In particular, we give a brief account of the technical sources of this paper, namely the works by Oort on infinitesimal deformations of abelian varieties with endomorphism $(8,[9])$, which we develop in detail, for the case of a cyclic automorphism, in 4 and in section 2 of the present article, the work of Bennama and Bertin ([1]) on extremal p.p.a.v with nontrivial automorphism and the so-called "degeneration argument", which permits ensuring the existence of products of extremal abelian varieties in each variety $A_{g}(p, \alpha)$.

The degeneration argument is based on the construction of abelian varieties with a given complex multiplication type ([1], [7]). Another important fact used throughout is the classification of the integer representations of cyclic groups of prime order ([3], [10]).

This section is almost completely expository, and the reader with some acquaintance with our previous papers $([4,[5,6])$ and the previously explained technical sources could safely skip its reading and come back occasionally to it when some justification is needed. The proofs included are only intended to cover some gaps in the argumentation or to correct some small inaccuracies in our preceding works.

In section 3 we prove Theorem 3.1, which states implication i) $\Rightarrow$ ii) of Theorem 1.1. Notice that $A_{g}(\alpha)$ is not defined in terms of total automorphism groups, but in terms of cyclic automorphisms of order $p$ acting on abelian varieties; therefore equalities of the types $A_{g}(\alpha)=A_{g}(\beta)$ cannot be discarded a priori. The implication $i) \Rightarrow i i$ ) consists precisely of proving the impossibility of such an equality assuming that $A_{g}(\alpha)$ is an irreducible component of $\operatorname{Sing} A_{g}$. Maybe a little bit surprisingly, this implication is by no means trivial.

As a preparatory step we also prove:

Lemma 3.2. Let $[X] \in A_{g}(p, \alpha)$ be a general element and $G_{+}=\operatorname{Aut}_{+}(X)$. If $d \alpha$ acts with $n_{0}=0$ and $\alpha \in Z\left(G_{+}\right)$, then $G_{+}$is abelian.

We hope that this lemma can shed some light on the problem of determining what finite groups can appear as the automorphism group of an abelian variety.

Section 4 is essentially devoted to proving Theorem 4.2 (implication iii) $\Rightarrow$ ii) in Theorem 1.1). The proof of the theorem is based on the study of the restriction of the automorphism group $G$ of a general element of $A_{g}(p, \alpha)$ to the automorphism group of a product of extremal abelian varieties $Z=X_{1} \times \ldots \times X_{r} \in A_{g}(\alpha)$. Lemma 4.1 implies that the image of this restriction in fact lies in $\prod_{i} \operatorname{Aut}\left(X_{i}\right)$, and then we can use the classification of $\operatorname{Aut}\left(X_{i}\right)$ for extremal abelian varieties established by Bennama and Bertin.

This is probably the most useful of our results for practically determining when an irreducible variety $A_{g}(p, \alpha)$ is an irreducible component of $\operatorname{Sing} A_{g}$. Indeed, there exists an exact sequence:

$$
1 \rightarrow C_{G_{+}}(\alpha) \rightarrow N_{G_{+}}(\langle\alpha\rangle) \rightarrow \Delta \rightarrow 1
$$


where the group $\Delta \subset \mathbb{F}_{p}^{*}$ is defined as follows ([1], [4): Let $d \alpha=\left\{\xi^{n_{1}}, \ldots, \xi^{n_{g}}\right\}$ and let $C$ be the set $\left\{n_{1}, \ldots, n_{g}\right\}$ considered as a subset of $\mathbb{F}_{p}^{*}$. Then, define $\Delta=\{k \in$ $\left.\mathbb{F}_{p}^{*} \mid k C=C\right\}$.

Computation of the group $\Delta$ is a trivial matter, and if for a fixed $\alpha, \Delta$ turns out to be trivial, then following Theorem 4.2 it only remains to prove that the centralizer $C_{G}(\langle\alpha\rangle)$ equals $\langle\alpha\rangle$ in order to conclude that $A_{g}(\alpha)$ is an irreducible component.

The advantage of working with $C_{G}(\alpha)$ instead of $N_{G}(\langle\alpha\rangle)$ is that there are important constraints for the structure of the centralizer:

Proposition 4.3. Consider a variety $A_{g}(p, \alpha)$ with $|\alpha|=p$ a prime odd number and $n_{0}=0$. Then, $\left|C_{G}(\alpha)\right|=p^{l} 2^{k}$ for some integers $l, k \geq 1$.

Thus, for each concrete case we are reduced to ruling out the existence of either involutions or elements of order $p$ not in $\langle\alpha\rangle$ and commuting with $\alpha$. Section 5 illustrates in detail how this analysis can be carried out in the case of abelian varieties of dimension 4 admitting an order 5 automorphism.

Almost all our statements are restricted to prime numbers different from 2. In fact, the study of varieties $A_{g}(\sigma)$ with $\sigma$ an involution seems to require different techniques and deserves a separated analysis.

\section{Preliminary Results}

The exposition in this section is a summary of results from [1] or [4. In a couple of places we indicate rather obvious generalizations.

2.1 Local moduli. Given a p.p.a.v. $X$ admitting an automorphism $\alpha \in \operatorname{Aut}(X)$ of order $p$, the analytical representation

$$
d \alpha: T_{0} X \rightarrow T_{0} X
$$

determines a decomposition of $T_{0} X$ as the direct sum of eigenspaces. In a suitable basis this map can be expressed as a diagonal matrix:

$$
d \alpha=\operatorname{diag}\left(I_{n_{0}}, \xi I_{n_{1}}, \ldots ., \xi^{p-1} I_{n_{p-1}}\right),
$$

with $\xi$ standing for a $p$-primitive root of 1 and $I_{n}$ denoting the identity $n \times n$ matrix.

In [8] and 9] Oort developed the theory of local deformation for polarized abelian varieties and polarized abelian varieties with endomorphisms. In particular, he proved the pro-representability of the functor $\mathcal{A}_{g}$ of local deformations of a polarized abelian variety in the category of Artinian algebras, the algebra pro-representing this functor is $\mathcal{O}=\mathbb{C}\left[\left[t_{i j}\right]\right]_{i \leq j}([\underline{8})$. This point of view has several advantages; for instance, if we define the functor $\mathcal{A}_{g}(\alpha)$ of local deformations associated with a pair $(X, \alpha)$, where $\alpha \in \operatorname{Aut}(X)$ is an order $p$ automorphism, then this functor is naturally a subfunctor: $\mathcal{A}_{g}(\alpha) \subset \mathcal{A}_{g}$. Thus, if $\mathcal{A}_{g}(\alpha)$ is pro-representable by a complete algebra $\mathcal{O}(\alpha)$, then this algebra must be a quotient of $\mathcal{O}$. This is indeed the case and we have that $\mathcal{O}(\alpha)=\mathcal{O} / \mathcal{I}_{\alpha}$, where $\mathcal{I}_{\alpha}$ is the ideal generated by

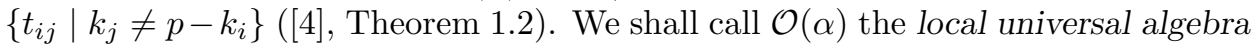
associated with $(X, \alpha)$.

More explicitly, the equations defining the local variety associated with our functor are given by the relations

$$
d \alpha T d \alpha^{t}=T
$$


with $T$ the symmetric matrix with entries $\left(t_{i j}\right)$. This relation is essentially expressing the fact that the automorphism $\alpha$ preserves the polarization of $X$. Moreover, since $d \alpha$ is a unitary matrix we have, equivalently,

$$
d \alpha T=T \overline{d \alpha}
$$

with - standing for complex conjugation. From this relation and the previous diagonal expression for $d \alpha$, the ideal $\mathcal{I}_{\alpha}$ is readily computed.

Thus, if we write the matrix of indeterminates $T_{\alpha}=\left(t_{i j}\right) \bmod \mathcal{I}_{\alpha}$ and introduce the notation $N_{l}=n_{0}+n_{1}+\ldots+n_{l}$ (with the convention $N_{-1}=N_{p+1}=0$ ), we obtain, combining the description of $\mathcal{O}(\alpha)$ with the previous diagonal representation of $d \alpha$, a block expression for $T_{\alpha}$ :

Lemma 2.1. Let $\mathcal{O}(\alpha)=\mathcal{O} / \mathcal{I}_{\alpha}$ be the local universal algebra associated with a pair $(X, \alpha)$. Then, in a suitable basis, the matrix $T_{\alpha}=T \bmod \mathcal{I}_{\alpha}$ admits an expression

$$
T=\left(T_{l}\right)
$$

having nonzero coefficients only in the blocks

$$
T_{0}=\left(t_{i j}\right)_{1 \leq i, j \leq n_{0}}, \quad T_{l}=\left(t_{N_{l-1}+i, N_{p-l-1}+j}\right)_{1 \leq i \leq n_{l}, 1 \leq j \leq n_{p-l}} \quad 1 \leq l \leq p-1 .
$$

We write $T_{l}=0$ in the previous expression if for some index $l$, the interval defining $T_{l}$ is empty.

Remark. Similar considerations apply for the infinitesimal deformation space for the cyclic subgroup of automorphisms generated by an element $\beta$ of arbitrary order. The relation

$$
d \beta T=T \overline{d \beta}
$$

is again the key point. This will be used in the proof of Theorem 3.1.

2.2 The varieties $A_{g}(\alpha)$. Let $X$ be a principally polarized abelian variety admitting an automorphism $\alpha$ of prime order $p$. Let us denote by $\alpha_{n}$ the representation of $\alpha$ in the $n$-torsion subgroup of $X$. It was proved in [4] that, for $n \geq 3$, an irreducible subvariety $A_{g}\left(p, \alpha_{n}\right) \subset A_{g}$ exists whose points are in bijection with abelian varieties $X$ admitting an automorphism of order $p$ and with $n$-torsion representation equivalent to that given by $\alpha$. This variety is obtained as a quotient of a subvariety $A_{g, n}\left(p, \alpha_{n}\right) \subset A_{g, n}$ of the moduli space of p.p.a.v. with an $n$-level structure. $A_{g, n}\left(p, \alpha_{n}\right)$ turns out to be a fine moduli space for varieties with an action of $\alpha$. This fact, combined with the pro-representability of the associated local functor, allows us to compute the dimension of $A_{g}\left(p, \alpha_{n}\right)$ as the Krull dimension of $\mathcal{O}(\alpha)$ and to obtain (same notation as in section 2.1):

$$
\operatorname{dim} A_{g}\left(p, \alpha_{n}\right)=\frac{n_{0}\left(n_{0}+1\right)}{2}+\sum_{i=1}^{(p-1) / 2} n_{i} \cdot n_{p-i}
$$

([4, after Theorem 1.2).

In what follows, in order to simplify the notation, we will denote such a variety by $A_{g}(\alpha)$ implicitly recognizing that the order of the automorphism $\alpha$ is fixed, as well as its entire and analytical representation type.

Therefore from the results of [4], the problem of determining the irreducible components of Sing $A_{g}$ reduces to determining what inclusions $A_{g}(\alpha) \subset A_{g}(\beta)$ could occur. 
For any pair $(X, \alpha)$ of a p.p.a.v. of dimension $g$ and an order $p$ automorphism acting on $X$ there exists a number $r$ such that $g=n_{0}+((p-1) / 2) r$ and $n_{i}+n_{p-i}=r$ for all $1 \leq i \leq p-1$ (迎, Proposition 2.1). Now, we state a slight generalization of Proposition 2.2 in [4]:

Lemma 2.2. Assume we have an inclusion $A_{g}(\alpha) \subset A_{g}(\beta)$ with $|\alpha|=p$ and $|\beta|=q, q>2$. Let $g=n_{0}+((p-1) / 2) r$ and $g=m_{0}+((q-1) / 2) s$ be the expressions associated to the eigenspaces of $d \alpha$ and $d \beta$. Then $n_{0} \leq m_{0}$ and $q \leq p$. Moreover, if $A_{g}(\alpha) \subset A_{g}(\beta)$ and $|\alpha|=2$, then $|\beta|=2$.

The proof, being essentially the same as in Proposition 2.2 in [4, is included here only as a sketch, and with some small inaccuracies corrected, for the reader's convenience.

Proof. If $\operatorname{dim} A_{g}(\alpha)=0$, then $[X]=A_{g}(\alpha)$ is a product $X=X_{1} \times \ldots \times X_{r}$ of extremal abelian varieties $X_{i}$ of dimension $\frac{p-1}{2}$. In this case the lemma easily follows from the results in 1 .

So, we assume that $\operatorname{dim} A_{g}(\alpha)>0$. Let $X_{0}$ be a general point in $A_{g}(\alpha)$. Choose a basis of $T_{0} X_{0}$ in which $d \alpha=\operatorname{diag}\left(\xi^{k_{i}}\right)$ is diagonal (the eigenvalues not necessarily in the order introduced in section 2.1). Let $\mathcal{I}_{\alpha}$ and $\mathcal{I}_{\beta}$ be the ideals in $\mathcal{O}=\mathbb{C}\left[\left[t_{i j}\right]\right]$ defining the local deformation spaces associated to $\alpha$ and $\beta$.

The inclusion $A_{g}(\alpha) \subset A_{g}(\beta)$ implies that $\mathcal{I}_{\beta} \subset \mathcal{I}_{\alpha}$, and, consequently, the equations

$$
d \beta T_{\alpha}=T_{\alpha} \overline{d \beta}
$$

with $T_{\alpha}=T \bmod \mathcal{I}_{\alpha}$ hold in $\mathcal{O} / \mathcal{I}_{\alpha}$.

Since $\operatorname{dim} A_{g}(\alpha)>0$, some $t_{i j}$ is not included in $\mathcal{I}_{\alpha}$. Here some different cases must be analyzed. Assume, for instance, that $t_{11} \notin \mathcal{I}_{\alpha}$, which is equivalent to saying that $k_{1}=0$. Then, (2.1) implies that

$$
d \beta=\left(\begin{array}{ccc}
a_{11} & & 0 \\
0 & A &
\end{array}\right)
$$

therefore $a_{11}=\bar{a}_{11}$. Thus $a_{11}$, being an eigenvalue of $d \beta$, must be equal to 1 .

Similarly if $t_{12} \notin \mathcal{I}_{\alpha}$, which is equivalent to $k_{1}=p-k_{2}$, then $t_{12} \notin \mathcal{I}_{\beta}$, since $\mathcal{I}_{\beta} \subset \mathcal{I}_{\alpha}$.

From equation (2.1) it follows that

$$
d \beta=\left(\begin{array}{cc}
\left(\begin{array}{ll}
a_{11} & a_{12} \\
a_{21} & a_{22}
\end{array}\right) & 0 \\
0 & A
\end{array}\right)
$$

and

We obtain:

$$
\left(\begin{array}{ll}
a_{11} & a_{12} \\
a_{21} & a_{22}
\end{array}\right)\left(\begin{array}{ll}
t_{11} & t_{12} \\
t_{12} & t_{22}
\end{array}\right)=\left(\begin{array}{ll}
t_{11} & t_{12} \\
t_{12} & t_{22}
\end{array}\right)\left(\begin{array}{ll}
\bar{a}_{11} & \bar{a}_{12} \\
\bar{a}_{21} & \bar{a}_{22}
\end{array}\right)
$$

$$
a_{11}=\overline{a_{11}}, a_{12}=\overline{a_{21}}=0 \text { and } a_{22}=\overline{a_{22}} .
$$

Thus,

$$
\left(\begin{array}{ll}
a_{11} & a_{12} \\
a_{21} & a_{22}
\end{array}\right) \in M_{2 \times 2}(\mathbb{R}) ;
$$

that is, its eigenvalues are complex conjugates.

In this way, we have proved that, for each eigenvalue of $d \alpha$ equal to 1 corresponds an eigenvalue of $d \beta$ equal to 1 , and to each pair of complex conjugate eigenvalues 
of $d \alpha$ corresponds a pair of complex conjugate eigenvalues of $d \beta$. This implies that $n_{0} \leq m_{o}$ and $s \geq r$. It follows also that $p \leq q$. The case $q=2$ can be treated similarly.

2.3 The degeneration argument. Consider an irreducible variety $A_{g}(\alpha)$. Assume that in the associated decomposition of tangent spaces $n_{0}=0$. Recall from 4], Lemma 2.7, that such a variety $A_{g}(\alpha)$ contains points representing abelian varieties of the form $Z=\left(X_{1} \times \ldots \times X_{r}, \Theta_{Z}\right)$, where the polarization is given by $\Theta_{Z}=\pi_{1}^{*} \Theta_{1}+\ldots+\pi_{r}^{*} \Theta_{r}$ with $\Theta_{i}$ the polarization of $X_{i}$. Each $X_{i}$ is of dimension $(p-1) / 2$ and admits an order $p$ automorphism, i.e., is an extremal abelian variety. As some of the various factors in the previous product could be isomorphic, we can write

$$
Z=X_{1}^{s_{1}} \times \ldots \times X_{t}^{s_{t}}, \quad s_{1}+\ldots+s_{t}=r
$$

with $X_{i} ¥ X_{j}$ if $i \neq j$.

If $\left(X_{\xi}, \Theta_{\xi}\right)$ is a generic point in $A_{g}(\alpha)$ and $G=\operatorname{Aut}\left(X_{\xi}, \Theta_{\xi}\right)$, then there exists an injective homomorphism of groups,

$$
G \hookrightarrow \operatorname{Aut}\left(Z, \Theta_{Z}\right),
$$

since the action of $G$ extends under specialization.

The following isomorphism was stated and used in 4. We include it here as a lemma and give a proof.

\section{Lemma 2.3.}

$$
\operatorname{Aut}\left(Z, \Theta_{Z}\right) \simeq\left[S_{s_{1}} \ltimes \operatorname{Aut}\left(X_{1}, \Theta_{1}\right)^{s_{1}}\right] \times \ldots \times\left[S_{s_{t}} \ltimes \operatorname{Aut}\left(X_{t}, \Theta_{t}\right)^{s_{t}}\right] .
$$

Proof. First of all, notice that if $X_{1}$ and $X_{2}$ are not isomorphic, then $\operatorname{Aut}\left(X_{1} \times\right.$ $\left.X_{2}\right)=\operatorname{Aut}\left(X_{1}\right) \times \operatorname{Aut}\left(X_{2}\right)$. Indeed, if $\pi_{i}$ denote the projections onto the factors, then $\pi_{1}^{*} \Theta_{1}+\pi_{2}^{*} \Theta_{2}$ being the polarization on $X_{1} \times X_{2}$ we must have

$$
\alpha^{*}\left(\pi_{1}^{*} \Theta_{1}+\pi_{2}^{*} \Theta_{2}\right)=\alpha^{*} \pi_{1}^{*}\left(\Theta_{1}\right)+\alpha^{*} \pi_{2}^{*}\left(\Theta_{2}\right)=\pi_{1}^{*} \Theta_{1}+\pi_{2}^{*} \Theta_{2} ;
$$

thus, $\alpha^{*}\left(\pi_{i}^{*} \Theta_{i}\right)=\pi_{i}^{*} \Theta_{i}$.

It remains to prove that if $X$ is an extremal abelian variety, then $\operatorname{Aut}\left(X^{n}\right) \simeq S_{n} \ltimes$ $\operatorname{Aut}(X)^{n}$. Assume that $\left(X_{1}, \Theta_{1}\right),\left(X_{2}, \Theta_{2}\right), \ldots,\left(X_{n}, \Theta_{n}\right)$ are isomorphic principally polarized extremal abelian varieties and let $\alpha \in \operatorname{Aut}\left(X_{1} \times X_{2} \times \ldots \times X_{n}\right)$. Arguing as before we have

$$
\alpha^{*}\left(\pi_{1}^{*} \Theta_{1}+\ldots+\pi_{n}^{*} \Theta_{n}\right)=\alpha^{*}\left(\pi_{1}^{*} \Theta_{1}\right)+\ldots+\alpha^{*}\left(\pi_{n}^{*} \Theta_{n}\right)=\pi_{1}^{*} \Theta_{1}+\ldots+\pi_{n}^{*} \Theta_{n} ;
$$

we conclude that $\alpha$ acts like an element of $S_{n}$ on the factors.

By standard arguments it is known that the locus of points

$$
U_{g}(\alpha)=\left\{\left[\left(X, \Theta_{X}\right)\right] \in A_{g}(\alpha) \mid \operatorname{Aut}\left(X, \Theta_{X}\right)=G\right\}
$$

is an open subscheme of $A_{g}(p, \alpha)$. Points in $U_{g}(\alpha)$ will be called "general points" of $A_{g}(\alpha)$.

Summarizing, for $[(X, \Theta)]$ general in $A_{g}(\alpha)$ there is an inclusion of groups,

$$
G=\operatorname{Aut}\left(X, \Theta_{X}\right) \subset\left[S_{s_{1}} \ltimes \operatorname{Aut}\left(X_{1}, \Theta_{1}\right)^{s_{1}}\right] \times \ldots \times\left[S_{s_{t}} \ltimes \operatorname{Aut}\left(X_{t}, \Theta_{t}\right)^{s_{t}}\right],
$$

for all possible choices of $\left(Z, \Theta_{Z}\right)$.

Note that $\prod_{i} \operatorname{Aut}\left(X_{i}, \Theta_{i}\right)^{s_{i}}$ is a normal subgroup of $\operatorname{Aut}\left(Z, \Theta_{Z}\right)$. 


\section{Characterization of the irreducible components of Sing $A_{g}$}

This section is devoted to characterizing irreducible components of the singular locus of $A_{g}$ in terms of the automorphism group of the general element of varieties $A_{g}(\alpha)$, assuming that in the corresponding decomposition $n_{0}=0$. The main result is:

Theorem 3.1. Let $A_{g}(\alpha)$ be a variety associated with an odd prime order automorphism $\alpha$ and acting with $n_{0}=0$. Then $A_{g}(\alpha)$ is an irreducible component of Sing $A_{g}$ if and only if for general $[X] \in A_{g}(\alpha)$, $\operatorname{Aut}_{+}(X) \simeq \mathbb{Z}_{p}$.

We start by proving a lemma giving important information about the structure of the automorphism groups of the general element of $A_{g}(\alpha)$.

Lemma 3.2. Let $[X] \in A_{g}(\alpha)$ be a general element and $G_{+}=\operatorname{Aut}_{+}(X)$. If $d \alpha$ acts with $n_{0}=0$ and $\alpha \in Z\left(G_{+}\right)$, then $G_{+}$is abelian.

Proof. We can assume that $G_{+} \neq\langle\alpha\rangle$; otherwise nothing needs to be proved. Let $\beta \in G_{+}$be any element such that $\langle\alpha\rangle \cap\langle\beta\rangle=1$. In this way $A_{g}(\alpha) \subset A_{g}(\beta)$. Consider the analytical representation of $\alpha$ and $\beta$ in $T_{0} X$. As the representation of $G$ in $T_{0} X$ is faithful, $[d \alpha, d \beta]=0$. Thus, there exists some basis of $T_{0} X$ in which both $d \alpha$ and $d \beta$ are represented as diagonal matrices. Assume, without loss of generality, that in that basis:

$$
\begin{aligned}
d \alpha & =\operatorname{diag}\left(\xi I_{n_{1}}, \ldots ., \xi^{p-1} I_{n_{p-1}}\right) \\
& =\operatorname{diag}\left(\xi^{k_{i}}\right)_{1 \leq i \leq g} .
\end{aligned}
$$

Denote by $\mathcal{O}(\alpha)$ the local universal algebra associated with the automorphism $\alpha$. This algebra is isomorphic to $\mathbb{C}\left[\left[t_{i j}\right]\right]_{i \leq j} / \mathcal{I}_{\alpha}$, with $\mathcal{I}_{\alpha}$ the ideal generated by $\left\langle t_{i j} \mid k_{j} \neq p-k_{i}\right\rangle$ (see section 2.1). According to Lemma 2.1 the matrix $T_{\alpha}=\left(t_{i j}\right)$ $\bmod \mathcal{I}_{\alpha}$ has a block expression:

$$
T_{\alpha}=\left(T_{l}\right)
$$

The inclusion $A_{g}(\alpha) \subset A_{g}(\beta)$ is characterized by the fact that the equation

$$
d \beta T=T \bar{d} \beta
$$

defines the trivial ideal in $\mathcal{O}(\alpha)$.

We claim that these relations imply that

$$
d \beta=\operatorname{diag}\left(\eta_{1} I_{n_{1}}, \eta_{2} I_{n_{2}}, \ldots, \eta_{n_{p-1}} I_{n_{p-1}}\right),
$$

with $\eta_{i}=\bar{\eta}_{p-i}$.

Let us develop, for instance, the case $l=1$. The matrix $T$ has an expression:

$$
T=\left(\begin{array}{cc}
0 & T_{1} \\
A & B
\end{array}\right)
$$

$T_{1}=\left(t_{i j}\right), 1 \leq i \leq n_{1}, N_{p-2}+1 \leq j \leq n_{p-1}$. Thus, if $d \beta=\operatorname{diag}\left(\eta_{1}, \ldots, \eta_{g}\right)$, then the resulting equations

$$
\operatorname{diag}\left(\eta_{1}, \ldots, \eta_{g}\right) T_{1}=T_{1} \operatorname{diag}\left(\overline{\eta_{N_{p-2}+1}}, \ldots, \overline{\eta_{g}}\right)
$$

clearly imply that $\eta_{1}=\ldots=\eta_{n_{1}}=\overline{\eta_{N_{p-2}+1}}=\ldots=\overline{\eta_{g}}$. Arguing similarly with each block $T_{l}$ the claim follows.

Finally, let $\gamma$ be any other element of $G_{+}$. As $\alpha$ is a nontrivial element of $Z\left(G_{+}\right)$, then

$$
d \gamma=\operatorname{diag}\left(D_{n_{1}}, \ldots, D_{n_{p-1}}\right)
$$


for some $l \times l$ diagonal blocks $D_{l}$. Matrices of the form $\lambda I$ commute with any matrix; thus we also have $[d \beta, d \gamma]=0$. Since the analytical representation of $G_{+}$is faithful and $\gamma, \beta$ can be chosen arbitrarily in the previous argument, we conclude that $G_{+}$is an abelian group.

Now we can proceed with the proof of the theorem.

Proof of Theorem 3.1. One of the two implications is obvious: it follows from 4 that the failure to be an irreducible component is associated with an inclusion $A_{g}(\alpha) \subset A_{g}(\beta)$. Therefore, if $\operatorname{Aut}_{+}(X)=\mathbb{Z}_{p}$ for general $[X] \in A_{g}(\alpha)$, then $A_{g}(\alpha)$ must be an irreducible component of $\operatorname{Sing} A_{g}$.

Conversely, assume that $A_{g}(\alpha)$ is an irreducible component of $\operatorname{Sing} A_{g}$. Let $[X] \in A_{g}(\alpha)$ be a general element and denote $G_{+}=\operatorname{Aut}_{+}(X)$. Assume $\left|G_{+}\right|>p$ (otherwise $G_{+} \simeq \mathbb{Z}_{p}$ and there is nothing to be proved). Let $\beta \in G_{+}-\langle\alpha\rangle$ be an element of prime order $q,|\beta|=q$. Since $[X]$ is general in $A_{g}(\alpha)$ and it is an irreducible component of $\operatorname{Sing} A_{g}$, we must have a double inclusion:

$$
A_{g}(\alpha) \subset A_{g}(\beta) \text { and } A_{g}(\beta) \subset A_{g}(\alpha) \text {. }
$$

Thus, using Lemma 2.2 (and the notation therein), we have that $m_{0}=n_{0}=0$ and $p=q$. We obtain as a first conclusion that $p$ is the unique prime divisor of $\left|G_{+}\right|$. Thus $G_{+}$is a $p$-group.

In particular, the center $Z\left(G_{+}\right)$of $G_{+}$contains an element of order $p$. Since for any element $\alpha^{\prime} \in G_{+}$of order $p, A_{g}(\alpha)=A_{g}\left(\alpha^{\prime}\right)$, we can assume without loss of generality that $\alpha \in Z\left(G_{+}\right)$. Using Lemma 3.2 we conclude that $G_{+}$is an abelian $p$-group.

Next we claim that $Z\left(G_{+}\right)=\langle\alpha\rangle$, which would conclude the proof. Assume that $\beta$ is another element of order $p$ commuting with $\alpha$. Consider the action of $\alpha$ on $E_{\mathbb{Q}}=E \otimes_{\mathbb{Z}} \mathbb{Q}$, with $E$ standing for the lattice defining $X\left(X \simeq \mathbb{C}^{g} / E\right)$.

Then we have $E_{\mathbb{Q}} \simeq \mathbb{Q}(\xi)^{\oplus r}$, where $\xi$ is a primitive $p$-root of 1 and $g=\frac{r(p-1)}{2}$. The action of $\alpha$ on $E_{\mathbb{Q}}$ determines a $\mathbb{Q}(\xi)$-linear automorphism of $E_{\mathbb{Q}}$.

Since $\beta$ is in the centralizer $C_{G}(\alpha), \alpha$ and $\beta$ can be simultaneously represented as linear automorphisms of $E_{\mathbb{Q}}$ as diagonal matrices of order $p$, say,

$$
\begin{gathered}
\alpha=\operatorname{diag}\left(\xi^{k_{1}}, \ldots, \xi^{k_{r}}\right), \\
\beta=\operatorname{diag}\left(\xi^{l_{1}}, \ldots, \xi^{l_{r}}\right) .
\end{gathered}
$$

$E_{\mathbb{Q}}$ is embedded into $\mathbb{C}^{g}$ by means of a map:

$$
\begin{gathered}
\mathbb{Q}(\xi)^{\oplus r} \hookrightarrow \mathbb{C}^{g}, \\
(\xi, \xi, \ldots, \xi) \rightarrow\left(\xi^{k_{11}}, \ldots, \xi^{k_{1,(p-1) / 2}}, \ldots, \xi^{k_{r 1}}, \ldots, \xi^{k_{r,(p-1) / 2}}\right),
\end{gathered}
$$

where the sets $C_{i}=\left\{k_{i j}\right\}_{1 \leq j \leq(p-1) / 2}$ are CM-types $([4])$.

In this way, the analytical representations of the automorphisms $\alpha$ and $\beta$ are given in $\mathbb{C}^{g}$ by

$$
\begin{gathered}
d \alpha=\operatorname{diag}\left(\xi^{k_{i} k_{i j}}\right), \\
d \beta=\operatorname{diag}\left(\xi^{l_{i} k_{i j}}\right) .
\end{gathered}
$$

Note that $C_{i}{ }^{\prime}:=\left\{k_{i} k_{i j}\right\}_{1 \leq j \leq(p-1) / 2}$ are still CM-types. Thus, for each $i$ we have that either $C_{1}{ }^{\prime} \cap C_{i}{ }^{\prime} \neq \emptyset$ or $C_{1}{ }^{\prime} \cap \overline{C_{i}{ }^{\prime}} \neq \emptyset$, where $\overline{C_{i}{ }^{\prime}}$ denotes the set obtained by taking the inverse of each element of $C_{i}{ }^{\prime}$ in $\mathbb{F}_{p}^{*}$. In this way, we have, after a 
reordering if necessary, that for each $i$ there exists a $j$ such that $\xi^{k_{1} k_{1 j}}=\xi^{k_{i} k_{i j}}$ or $\xi^{k_{1} k_{1 j}}=\xi^{k_{i} \bar{k}_{i j}}$.

Now, $A_{g}(\alpha)=A_{g}(\beta)$ implies that if $\xi^{k_{1} k_{1 j}}=\xi^{k_{i} k_{i j}}$, then $\xi^{l_{1} k_{1 j}}=\xi^{l_{1} k_{i j}}$. This can be written as the system of congruences:

$$
\begin{gathered}
k_{1} k_{1 j} \equiv k_{i} k_{i j} \quad \bmod p, \\
l_{1} k_{1 j} \equiv l_{i} k_{i j} \quad \bmod p .
\end{gathered}
$$

Thus, $k_{i} / k_{1} \equiv l_{i} / l_{1} \bmod p$, where quotients mean taking inverses in the multiplicative group $\mathbb{F}_{p}^{*}$.

Similarly if $\xi^{k_{1} k_{1 j}}=\xi^{k_{i} \bar{k}_{i j}}$, then $\xi^{l_{1} k_{1 j}}=\xi^{l_{1} \bar{k}_{i j}}$, and we obtain also in this case that

$$
k_{i} / k_{1} \equiv l_{i} / l_{1} \bmod p .
$$

We conclude that in $\operatorname{End}\left(\mathbb{Q}(\xi)^{\oplus r}\right)$ the relation $\alpha^{-k_{1}}=\beta^{-l_{1}}$ or equivalently $\alpha^{p-k_{1}}=\beta^{p-l_{1}}$ holds; that is, $\alpha$ and $\beta$ generate the same cyclic subgroup of $G$.

Finally, assume that $|\beta|=p^{n}$, with $n>1$, commutes with $\alpha$. Since $\alpha$ and $\beta$ commute, $\beta$ induces a $\mathbb{Q}(\xi)$ - linear endomorphism of $\mathbb{Q}(\xi)^{\oplus r}$. Thus, in a suitable basis,

$$
\beta=\operatorname{diag}\left(q_{1}(\xi), \ldots, q_{r}(\xi)\right),
$$

with $q_{i}$ polynomials and $q_{i}(\xi)^{p^{n}}=1$. In this way we obtain

$$
\mathbb{Q}(\eta) \subset \mathbb{Q}(\xi),
$$

with $\eta$ some $p^{n}$-root of unity. Thus, $\eta$ must be a primitive $p$-root of 1 and $\beta$ is of order $p$. This final contradiction proves that $Z\left(G_{+}\right)=\langle\alpha\rangle$.

\section{The Characterization In terms of $N_{G}(\langle\alpha\rangle)$}

Let $A_{g}(\alpha)$ be, as before, an irreducible subvariety of $\operatorname{Sing} A_{g}$ corresponding to an automorphism of order $p>2$ and with $n_{0}=0$.

In what follows we make use of the results and notation of section 2.3. We consider an element $Z \in A_{g}(\alpha)$ of the form $Z=\prod_{i} X_{i}^{n_{i}}$, with each $X_{i}$ an extremal abelian variety, $X_{i}$ not isomorphic to $X_{j}$ if $i \neq j$.

Lemma 4.1. Let $G$ be the automorphism group of a general element of $A_{g}(\alpha)$, $\beta \in G$ an element of prime order $q>2$ and $[Z] \in A_{g}(\alpha)$ a product of extremal abelian varieties. Then the image of $\langle\beta\rangle$ in $\operatorname{Aut}(Z)$ is a subgroup of $\prod_{i} \operatorname{Aut}_{+}\left(X_{i}\right)^{n_{i}}$.

Proof. Denote by

$$
\phi: G \hookrightarrow \prod_{i}\left(\operatorname{Aut}\left(X_{i}\right)^{n_{i}} \rtimes S_{n_{i}}\right),
$$

the inclusion proved in 4], Lemma 2.10. We must prove that $\phi$ does not permute the factors.

Since $G$ is the automorphism group of a general element of $A_{g}(\alpha)$, the analytical spectrum of $\beta$ must be constant on elements of $A_{g}(\alpha)$. For $[X] \in A_{g}(\alpha)$, a general element, fix a decomposition into eigenspaces under the action of $d \beta$ :

$$
V=V_{0} \oplus V_{1} \oplus \ldots \oplus V_{s}
$$

with $\operatorname{dim} V_{0}=m_{0}, \operatorname{dim} V_{i}=\frac{q-1}{2}$ for $i>0, g=m_{0}+s \frac{q-1}{2}$ and $d \beta_{i}=\left.d \beta\right|_{V_{i}}$ defining a CM-type associated to $q$. Since

$$
\operatorname{tr}\left(d \beta_{i}\right)=\eta^{m_{1}}+\ldots+\eta^{\frac{m_{\frac{q-1}{2}}}{2}},
$$


for $i>0$ and $\eta$ a $q$-primitive root of 1 ,

$$
\operatorname{tr}\left(d \beta_{i}\right)+\operatorname{tr}\left(\bar{d}_{i}\right)=2 \operatorname{Re}\left(\operatorname{tr} d \beta_{i}\right)=1 .
$$

Therefore, we see that $\operatorname{Re}(\operatorname{tr} d \beta)=m_{0}+\frac{s}{2}$.

Since $d \beta$ acts like a block-diagonal matrix and $\phi$ permutes the blocks, we see that

$$
\operatorname{Re}(\operatorname{tr} \phi(d \beta)) \leq \operatorname{Re}(\operatorname{tr} d \beta)),
$$

with equality if and only if $\phi(\beta) \in \prod_{j}\left(\operatorname{Aut}\left(X_{j}\right)^{n_{j}}\right)$. Finally, if some block is sent under a projection to its additive inverse we get similarly that $(\operatorname{tr} \phi(d \beta)) \neq(\operatorname{tr}(d \beta))$. This shows that in fact $\phi(\beta) \in \prod_{j}\left(\operatorname{Aut}_{+}\left(X_{j}\right)\right)^{n_{j}}$.

Next we state the main theorem of this section, which gives another characterization of irreducible components of $\operatorname{Sing} A_{g}$.

Theorem 4.2. Let $[X] \in A_{g}(\alpha)$ be a general element. Then $G_{+}=\mathbb{Z}_{p}$ if and only if $\langle\alpha\rangle=N_{G_{+}}(\langle\alpha\rangle)$.

Proof. It is obvious that $G_{+}=\mathbb{Z}_{p}$ implies $\langle\alpha\rangle=N_{G_{+}}(\langle\alpha\rangle)$.

Assume that $\langle\alpha\rangle=N_{G_{+}}(\langle\alpha\rangle), C_{G_{+}}(\alpha) \subset N_{G_{+}}(\langle\alpha\rangle)$ implies $C_{G_{+}}(\alpha)=\langle\alpha\rangle$. We conclude that $\langle\alpha\rangle$ is a $p$-Sylow subgroup of $G_{+}$. Thus $\left|G_{+}\right|=p q$, with $p$ and $q$ relatively prime, and $q$ the number of $p$-Sylow subgroups of $G_{+}$.

Consider $[Z]=\left[X_{1} \times \ldots \times X_{r}\right] \in A_{g}(\alpha)$, with each $X_{i}, i>0$, extremal of dimension $\operatorname{dim} X_{i}=\frac{p-1}{2}$. Here some $X_{i}$ could be isomorphic to an $X_{j}$.

Let $\pi: G_{+} \rightarrow \operatorname{Aut}(Z)$ be the natural inclusion. Applying Lemma 4.1 we have that $\Pi^{-1}\left(\prod_{i}\right.$ Aut $\left._{+}\left(X_{i}\right)\right) \subset G_{+}$contains at least $(p-1) q$ elements; since $\left|G_{+}\right|=p q$ we conclude that $G_{+} \subset \Pi_{i}$ Aut $_{+}\left(X_{i}\right)$. Consider the projections

$$
\pi_{i}: G_{+} \rightarrow \operatorname{Aut}_{+}\left(X_{i}\right)
$$

Following [1, Theorems 1 and 2, there are two possibilities for the image of $\pi_{i}\left(G_{+}\right) \subset \operatorname{Aut}_{+}\left(X_{i}\right)$ : it could be either $\mathbb{Z}_{p} \rtimes \mathbb{Z}_{t}$, with $t$ a divisor of $\frac{p-1}{2}$ or $P S L_{2}\left(\mathbb{F}_{p}\right)$. Moreover, $P S L_{2}\left(\mathbb{F}_{p}\right)$ can appear only for $p>3$ and congruent with -1 modulo 4 . Denote $G_{i}=\pi_{i}\left(G_{+}\right)$and $K_{i}=\operatorname{ker} \pi_{i}$.

First, let us prove that $G_{i}$ cannot be isomorphic to $P S L_{2}\left(\mathbb{F}_{p}\right)$. Assume, for instance, that $G_{1}=P S L_{2}\left(\mathbb{F}_{p}\right)$. Let $\left|K_{1}\right|=k$. The group $K_{1}$ acts by conjugation on the set of $q p$-Sylow subgroups of $G_{+}$. Thus, the number of $p$-Sylow subgroups of $P S L_{2}\left(\mathbb{F}_{p}\right)$ must be $\frac{q}{k}$ and thus $q=k(l p+1)$ for some natural number $l$. It follows from the exact sequence

$$
1 \rightarrow K_{1} \rightarrow G_{+} \rightarrow P S L_{2}\left(\mathbb{F}_{p}\right) \rightarrow 1
$$

that $p q=k p \frac{\left(p^{2}-1\right)}{2}$, and so $l p+1=\frac{\left(p^{2}-1\right)}{2}$. Therefore we get the contradiction $3=p^{2}-2 l p$.

Finally, let $G_{i}=\mathbb{Z}_{p} \rtimes \mathbb{Z}_{t}$. Thus, $G_{i}$ has a unique $p$-Sylow subgroup and in consequence all the elements of order $p$ in $G_{+}$have their images contained in $\mathbb{Z}_{p}$. Since $G_{+}$has exactly $q p$-Sylow subgroups, $K_{i}$ contains at least $q$ elements. We conclude that $\left|K_{i}\right|=q$. Thus, $G_{i}=\mathbb{Z}_{p}$. Since $G_{+} \subset \prod_{i} G_{i}$ we conclude that $q=1$ and $G_{+}=\langle\alpha\rangle$.

In order to use Theorem 4.2 to determine when $A_{g}(\alpha)$ is an irreducible component of $\operatorname{Sing} A_{g}$ it is useful to introduce the exact sequence

$$
1 \rightarrow C_{G_{+}}(\alpha) \rightarrow N_{G_{+}}(\langle\alpha\rangle) \rightarrow \Delta \rightarrow 1,
$$


where the group $\Delta \subset \mathbb{F}_{p}^{*}$ is defined as follows (1], 4]): Let $d \alpha=\left\{\xi^{n_{1}}, \ldots, \xi^{n_{g}}\right\}$ and let $C$ be the set $\left\{n_{1}, \ldots, n_{g}\right\}$ considered as a subset of $\mathbb{F}_{p}^{*}$. Then, define $\Delta=\{k \in$ $\left.\mathbb{F}_{p}^{*} \mid k C=C\right\}$.

Thus, according to Theorem 4.2 , if $\Delta \neq i d$, then $A_{g}(\alpha)$ is not an irreducible component of $\operatorname{Sing} A_{g}$.

On the other hand, if $\Delta=1$ we cannot ensure that $A_{g}(\alpha)$ is an irreducible component, but we can try to deduce that $N_{G_{+}}(\langle\alpha\rangle)$ is equal to $\langle\alpha\rangle$ by studying the structure of the centralizer of $\alpha$. Some information can be gained if we apply the following:

Proposition 4.3. Consider a variety $A_{g}(\alpha)$ with $|\alpha|=p$ a prime odd number and $n_{0}=0$. Then, $\left|C_{G}(\alpha)\right|=p^{l} 2^{k}$ for some integers $l, k \geq 1$.

Proof. It is sufficient to prove that $C_{G}(\alpha)$ does not contain elements of prime order $q$ different from $p$ or 2 . The argument is similar to that employed in the last part of the proof of Theorem 3.1.

Assume there exists $\beta \in C_{G}(\alpha)$ such that $|\beta|=q$ with $q$ a prime number different from $p$ and 2. Considering $\alpha$ and $\beta$ as $\mathbb{Q}(\xi)$-endomorphisms of $E_{\mathbb{Q}}$ and arguing as in the last part of the proof of Theorem 3.1, we obtain

$$
\beta=\operatorname{diag}\left(q_{1}(\xi), \ldots, q_{r}(\xi)\right),
$$

with $q_{i}$ integer polynomials. Moreover, $\beta^{q}=1$ implies that $q_{k}(\xi)=\eta$ with $\eta$ a $q$-primitive root of 1 . Therefore we have

$$
\mathbb{Q}(\eta) \subset \mathbb{Q}(\xi),
$$

which is impossible ([12, Prop. 2.4).

Remark. In the next section we will provide a simple example of a variety $A_{g}(\alpha)$ for which $C_{G}(\alpha)$ contains both elements of order $p$ not in $\langle\alpha\rangle$ and elements of order 2 .

\section{EXAMPLE: $g=4$ AND $p=5$}

The goal of this section is to show how our previous results and methods can be used in practice on the example of $A_{4}(\alpha)$. Assuming $g=4$, let us analyze the varieties $A_{4}(\alpha)$ with $|\alpha|=5$ and $n_{0}=0$. In this case we must have $r=2$ and, therefore, the possible analytical spectra $\alpha_{0}, \alpha_{1}, \alpha_{2}$ are:

$$
d \alpha_{0}=\operatorname{diag}\left(\xi I_{2}, \xi^{2} I_{2}\right), \quad d \alpha_{1}=\operatorname{diag}\left(\xi, \xi^{2} I_{2}, \xi^{4}\right) \text { and } d \alpha_{2}=\operatorname{diag}\left(\xi, \xi^{2}, \xi^{3}, \xi^{4}\right),
$$

with $\xi^{5}=1$. Moreover, since $\mathbb{Z}[\xi]$ has an ideal class equal to 1 ([12, Thm. 11.1) the entire representation of every $\alpha$ is forced to be equivalent to $\mathbb{Z}[\xi]^{\oplus 2}$. Thus, for each analytical spectrum there is associated a unique irreducible variety $A_{4}\left(\alpha_{i}\right)$. The dimension of these varieties is given by $\operatorname{dim} A_{4}\left(\alpha_{i}\right)=i$.

Next, we analyze which of these could be an irreducible component of $\operatorname{Sing} A_{4}$.

We start with $A_{4}\left(\alpha_{0}\right)$. First of all note that $\operatorname{dim} A_{4}\left(\alpha_{0}\right)=0$ implies that $A_{4}\left(\alpha_{0}\right)=\left[X_{0} \times X_{0}\right]$ with $X_{0}$ a 2 -dimensional p.p.a.v. having an order 5 automorphism $\phi$. Thus, Aut $\left(X_{0} \times X_{0}\right)$ admits at least two different order 5 subgroups with commuting generators, namely $\langle(\phi, \phi)\rangle$ and $\left\langle\left(\phi, \phi^{2}\right)\right\rangle$. Assuming $d \phi=\operatorname{diag}\left(\xi, \xi^{2}\right)$ we see that $d\left(\phi, \phi^{2}\right)=\operatorname{diag}\left(\xi, \xi^{2}, \xi^{2}, \xi^{4}\right)$ and in consequence

$$
A_{4}\left(\alpha_{0}\right) \subset A_{4}\left(\alpha_{1}\right) \text {. }
$$


Moreover, $\gamma=\left(\phi^{2}, \phi^{3}\right)$ is an order 5 automorphism $\gamma \in \operatorname{Aut}\left(X_{0} \times X_{0}\right)$ with

$$
d \gamma=\operatorname{diag}\left(\xi^{2}, \xi^{4}, \xi^{3}, \xi\right),
$$

and $A_{4}\left(\alpha_{0}\right) \subset A_{4}\left(\alpha_{2}\right)$ as well.

Further, we can define another automorphism $\sigma \in \operatorname{Aut}_{+}\left(X_{0} \times X_{0}\right)$ of order 2 given by $\sigma=(i d,-i d)$.

The group $\Delta$ associated to the spectrum of $\alpha_{0}$ (see the exact sequence (4.1)) is trivial. Therefore, this simple example shows a variety $A_{g}(\alpha)$ with centralizer $C_{G}(\alpha)$ containing both elements of order $p$ not in $\langle\alpha\rangle$ and elements of order 2 (see Proposition 4.3).

Now, we analyze the variety $A_{4}\left(\alpha_{2}\right)$. For this, $\Delta \simeq \mathbb{F}_{5}^{*}$ and we can apply Theorem 4.2 in order to conclude that $A_{4}\left(\alpha_{2}\right)$ is not an irreducible component of $\operatorname{Sing} A_{4}$. Besides this general fact, we can explicitly find a variety $A_{4}(\sigma)$ with $|\sigma|=2$ such that

$$
A_{4}\left(\alpha_{2}\right) \subset A_{4}(\sigma) ;
$$

this can be done by considering the corresponding varieties in $\mathbb{H}_{4}$ (see [4], section 2.1 for notation). In fact, we can choose a lifting of $A_{4}\left(\alpha_{2}\right)$ as the subvariety

$$
\operatorname{Fix}\left(\alpha_{s}\right)=\mathbb{H}_{4}\left(\alpha_{s}\right)=\left\{Z \in \mathbb{H}_{4} \mid \alpha_{s} \star Z=Z\right\} \subset \mathbb{H}_{4}
$$

a calculation gives

$$
\operatorname{Fix}\left(\alpha_{s}\right)=\left(\begin{array}{cccc}
2\left(\tau_{1}+\tau_{2}\right) & 2 \tau_{1} & \tau_{1}+\tau_{2} & 2 \tau_{2} \\
2 \tau_{1} & 4 \tau_{1} & 3 \tau_{1}-\tau_{2} & \tau_{1}+\tau_{2} \\
\tau_{1}+\tau_{2} & 3 \tau_{1}-\tau_{2} & 4 \tau_{1} & 2 \tau_{1} \\
2 \tau_{2} & \tau_{1}+\tau_{2} & 2 \tau_{1} & 2\left(\tau_{1}+\tau_{2}\right)
\end{array}\right)
$$

If we consider $s \in G L(4, \mathbb{Z})$ given by

$$
s=\left(\begin{array}{llll}
0 & 0 & 0 & 1 \\
0 & 0 & 1 & 0 \\
0 & 1 & 0 & 0 \\
1 & 0 & 1 & 0
\end{array}\right)
$$

we have $\sigma_{s}^{-1} \circ \alpha_{s} \circ \sigma_{s}=\alpha_{s}^{4}$, and thus $\sigma_{s} \in N_{S p(8, \mathbb{Z})}(\langle\alpha\rangle)$. Then, $\operatorname{Fix}\left(\sigma_{s}\right)=\mathbb{H}_{4}\left(2, \sigma_{s}\right)$ determines a 6-dimensional component

$$
A_{4}\left(2, \sigma_{n}\right) \subset \operatorname{Sing} A_{4}
$$

Since $\sigma_{s}: \mathbb{H}_{4} \rightarrow \mathbb{H}_{4}$ given by $Z \rightarrow \sigma_{s} \star Z$ is a biholomorphism that is the identity on $\mathbb{H}_{4}\left(5, \alpha_{s}\right)$, we have $\mathbb{H}_{4}\left(5, \rho_{s}\right) \subset \mathbb{H}_{4}\left(2, \sigma_{s}\right)$.

In fact, we have

$$
\operatorname{Fix}\left(\sigma_{s}\right)=\left(\begin{array}{llll}
a & c & d & e \\
c & b & f & d \\
d & f & b & c \\
e & d & c & a
\end{array}\right)
$$

So far we have obtained that $A_{g}\left(\alpha_{0}\right) \subset A_{4}\left(\alpha_{1}\right) \cap A_{4}\left(\alpha_{2}\right)$ and $A_{4}\left(\alpha_{2}\right)$ is not an irreducible component of $\operatorname{Sing} A_{4}$, contained in $A_{4}(\sigma)$ with $\sigma$ of order two.

Finally, we consider $A_{4}\left(\alpha_{1}\right)$ and prove that it is an irreducible component of $\operatorname{Sing} A_{4}$. First, observe that the group $\Delta$ associated to this spectrum is trivial. Thus, according to Theorem 4.2 and Proposition 4.3, the failure to be an irreducible component must be associated either with the existence in $C_{G_{+}}(\alpha)$ of automorphisms of order 5 not in $\langle\alpha\rangle$ or with an order 2 automorphism. 
Assume that $A_{4}\left(\alpha_{1}\right) \subset A_{4}\left(\alpha_{2}\right)$. The proof of Lemma 2.2 contains the basic principle that "to each pair of complex conjugate eigenvalues of $d \alpha_{1}$ there corresponds a pair of complex conjugate eigenvalues of $d \alpha_{2}$ ". Thus, if we fix a basis in which

$$
d \alpha_{1}=\operatorname{diag}\left(\xi, \xi^{2}, \xi^{2}, \xi^{4}\right),
$$

then in this basis $d \alpha_{2}$ must have in the first and in the last diagonal entry complex conjugate values. Assume, for instance, that

$$
d \alpha_{2}=\operatorname{diag}\left(\xi, \xi^{2}, \xi^{3}, \xi^{4}\right) .
$$

Then,

$$
d \alpha_{1} d \alpha_{2}=\operatorname{diag}\left(\xi^{2}, \xi^{4}, 1, \xi^{3}\right)
$$

but the number $n_{i}$ and $r$ associated to this spectrum cannot be expressed like $4=n_{0}+2 r$. The remaining possibilities can be computed easily to conclude that the inclusion $A_{4}\left(\alpha_{1}\right) \subset A_{4}\left(\alpha_{2}\right)$ is impossible.

Similar arguments prove that

$$
A_{4}\left(\alpha_{1}\right) \nsubseteq A_{4}(\alpha)
$$

even in the case when $\alpha$ is an order 5 automorphism with $n_{0}>0$ (and then necessarily $n_{0}=2$ ).

Last, consider the possibility that $A_{4}\left(\alpha_{1}\right) \subset A_{4}(\sigma)$ with $|\sigma|=2$ and $\alpha_{1} \sigma=\sigma \alpha_{1}$ in $G$. Once again, the proof of Lemma 2.2 implies that if $d \alpha_{1}=\operatorname{diag}\left(\xi, \xi^{2}, \xi^{2}, \xi^{4}\right)$, then in the same basis $d \sigma=\operatorname{diag}\left(1, \sigma_{1}, \sigma_{2}, 1\right)$ with either $\sigma_{1}=\sigma_{2}=-1$ or $\sigma_{1} \sigma_{2}=-1$. We now specialize to the product $\left[X_{0} \times X_{0}\right]$ in order to conclude that $\operatorname{Aut}_{+}\left(X_{0}\right)$ must contain an automorphism of order 2 , which gives a contradiction, as $\operatorname{Aut}_{+}\left(X_{0}\right)=\mathbb{Z}_{5}$ (see [1] or [5]).

We conclude that $A_{4}\left(\alpha_{1}\right)$ is an irreducible component of $\operatorname{Sing} A_{4}$.

\section{REFERENCES}

1. H. Bennama and J. Bertin, Remarques sur les variétés abéliennes avec un automorphisme d'ordre premier, Manuscripta Math. 94 (1997), 409-425. MR.1484635 (99e:14050)

2. C. Birkenhake and H. Lange, Complex abelian varieties, Springer-Verlag (Second Edition), 2004. MR2062673 (2005c:14001)

3. C. W. Curtis, Representation theory of finite groups and associative algebras, Pure and Appl. Math., vol. XI, Interscience, 1962. MR0144979 (26:2519)

4. V. González-Aguilera, J. M. Muñoz-Porras and A. G. Zamora, On the irreducible components of the singular locus of $A_{g}$, Journal of Algebra 240 (2001), 230-250. MR.1830552 (2002b:14054)

5. V. González-Aguilera, J. M. Muñoz-Porras and A. G. Zamora, On the 0-dimensional irreducible components of the singular locus of $A_{g}$, Archiv der Mathematik 84 (2005), 298-303. MR.2135039 (2006b:14076)

6. V. González-Aguilera, J. M. Muñoz-Porras and A. G. Zamora, Some recent results on the irreducible components of the singular locus of $A_{g}$, Contemporary Mathematics 397, Amer. Math. Soc., 2006, 119-126. MR2218002 (2007b:14099)

7. D. Mumford, Abelian varieties, Oxford University Press, 1970. MR0282985 (44:219)

8. F. Oort, Finite group schemes, local moduli for abelian varieties and lifting problem, Algebraic Geometry, Oslo, 1970, Wolters-Noordhoff (1971), 265-296. MR0301026 (46:186)

9. F. Oort, Singularities of coarse moduli schemes, Sem. Dubreil, Lecture Notes in Math. 586, Springer, 1977, 61-76. MR.518011 (80e:14002)

10. I. Reiner, Integral representations of cyclic groups of prime order, Proc. Amer. Math. Soc. 8 (1957), 142-146. MR0083493 (18:717a) 
11. G. Shimura, Abelian varieties with complex multiplication and modular functions, Princeton University Press, 1998. MR,1492449 (99e:11076)

12. L. Washington, Introduction to cyclotomic fields (Second Edition), Grad. Texts in Math. 83, Springer-Verlag, 1997. MR.1421575 (97h:11130)

Departamento de Matemáticas, Universidad Técnica Federico Santa María, Casilla 110-V, Valparaíso, Chile

E-mail address: victor.gonzalez@usm.cl

Departamento de Matemáticas, Universidad de Salamanca, Plaza de la Merced 1-4, SAlamanca 37008 , Spain

E-mail address: jmp@usal.es

Departamento de Matemáticas, Universidad Autónoma de Zacatecas, Camino a la Bufa y Calzada Solidaridad, C.P. 98000, Zacatecas, Zac., México

E-mail address: alexiszamora06@gmail.com 